\title{
Technè
}

La science au service de l'histoire de l'art et de la préservation des biens culturels

40 | 2014

Thérapéia. Polychromie et restauration de la sculpture dans l'Antiquité

\section{Introduction. Étudier et conserver la polychromie antique. Vidéo-microscopie et archéologie de la \\ surface}

The study and conservation of ancient polychromy. Video microscopy and surface archaeology

\section{Brigitte Bourgeois}

\section{OpenEdition Journals}

Édition électronique

URL : https://journals.openedition.org/techne/2948

DOI : $10.4000 /$ techne. 2948

ISSN : 2534-5168

\section{Éditeur}

C2RMF

\section{Édition imprimée}

Date de publication : 26 novembre 2014

Pagination : 3-7

ISBN : 978-2-7118-6218-4

ISSN : $1254-7867$

\section{Référence électronique}

Brigitte Bourgeois, «Introduction. Étudier et conserver la polychromie antique. Vidéo-microscopie et archéologie de la surface », Technè [En ligne], 40 | 2014, mis en ligne le 24 juillet 2020, consulté le 10 février 2022. URL : http://journals.openedition.org/techne/2948; DOI : https://doi.org/10.4000/ techne.2948

\section{(c) $)(1)(9)$}

La revue Technè. La science au service de l'histoire de l'art et de la préservation des biens culturels est mise à disposition selon les termes de la Licence Creative Commons Attribution - Pas d'Utilisation Commerciale - Pas de Modification 4.0 International. 
Brigitte Bourgeois

\section{Étudier et conserver la polychromie antique. Vidéo-microscopie et archéologie de la surface}

\section{Introduction}

Résumé. On expose ici la conception d'un volume consacré au devenir matériel de la sculpture dans l'Antiquité, particulièrement aux soins (thérapéia) portés à entretenir l'éclat de la surface des effigies en Grèce ancienne. L'article rappelle l'apport des études en vidéo-microscopie dans une démarche d'archéologie historique de la couleur qui a permis de mettre en évidence des remaniements de surface antiques.

Mots-clés. Conservation-restauration, Antiquité, polychromie, dorure, Égypte, Grèce, Délos, Chypre, vidéo-microscopie, microscopie $3 D$ de surface.

Il y a quelques années, dans cette même revue, Marie Berducou consacrait un article aux prémices de la conservation et de la restauration dans l'Antiquité ${ }^{1}$. Elle rappelait, témoignages d'auteurs grecs et latins à l'appui, combien «le passé et le sacré sont concrètement omniprésents dans le paysage et le quotidien des sociétés antiques, et ce dans quoi ils s'incarnent fait l'objet d'un entretien vigilant et de soins constants ». Au vu de l'importance de ce "projet conservatoire » déjà réfléchi et mis en ouvre, elle nous invitait à repenser les limites de la périodisation traditionnellement établie qui tend à négliger ce chapitre.

En réponse en quelque sorte à son invite, j'ai voulu reprendre l'enquête dans un numéro consacré à la question de l'antiquité de la restauration dans le domaine de la sculpture. Le lecteur qui lira ce volume le refermera, je l'espère, avec la conviction qu'en effet un véritable socle, malheureusement englouti, sous-tend la «renaissance» des temps modernes et que tout essai de synthèse sur l'histoire de la restauration ne saurait faire l'impasse sur la période antique.

Le choix de la sculpture s'est imposé pour plusieurs raisons. C'est, en dehors de l'architecture qui n'entre pas dans le périmètre de Technè, le seul domaine de production qui permette de croiser des sources textuelles et des documents archéologiques suffisamment bien conservés. Cette catégorie a, de plus, joué un rôle essentiel dans le développement de pratiques conservatoires en raison de la fonction sacrée d'œuvres qui, au cœur des religions antiques, matérialisaient l'échange des hommes avec les dieux. On sait combien la recherche en sciences humaines réinterroge à l'heure actuelle les notions de figuration du divin et de captation de l'invisible
The study and conservation of ancient polychromy. Video microscopy and surface archaeology

Abstract. One explains about the editorial project of a volume on the conservation of sculpture in Antiquity, focusing on the care (therapeia) paid to maintaining the shine of surface treatments in ancient Greece. In allowing for the detection of physical remains of ancient repaints, video-microscopy has played an important role in the development of a historical archaeology of the surface.

Keywords. Conservation, Antiquity, polychromy, gilding, Egypt, Greece, Delos, Cyprus, video microscopy, 3D surface microscopy.

dans les sociétés antiques, et combien la polysémie d'un mot grec tel qu'agalma, traduit trop vite par «statue de culte», cache de complexité par rapport à nos façons modernes de penser la sculpture ${ }^{2}$. On connait aussi l'essor des travaux autour de la polychromie de la plastique grecque et romaine (fig. 1 a-b), porté par une communauté internationale ${ }^{3}$. On se souvient enfin que, depuis longtemps, des témoignages épars dans la littérature et dans l'épigraphie grecque avaient attiré l'attention des savants sur des pratiques visant à garder et à remettre en bon état non seulement l'intégrité de la forme sculptée, mais aussi l'éclat de la surface. Il m'a paru utile d'enrichir l'état de la question par l'apport de la restauration et du laboratoire, en faisant connaître plusieurs dossiers d'œuvres (notamment des trois départements d'antiquités du Louvre) récemment étudiées au C2RMF - tout en les réinsérant dans une perspective historique plus large. La contribution que propose ce numéro croise donc le champ des recherches sur la polychromie avec celui des études sur l'histoire de la restauration. Il regroupe et analyse un certain nombre de données relatives au devenir matériel de la sculpture dans l'Antiquité (Égypte, Grèce et Chypre), en privilégiant le domaine grec avec la question des soins («thérapéia»), de portée religieuse et profane indissociablement liée, apportés aux effigies.

Le rapport à l'objet diffère de l'ordinaire. En matière d'histoire de l'art, et particulièrement d'art grec, ce qui n'a cessé d'être exalté est le geste créateur; ce qui focalise le regard mental porté sur le passé est le désir de revoir la splendeur première de l'œuvre. Ici, c'est l'inverse. Le numéro porte sur 


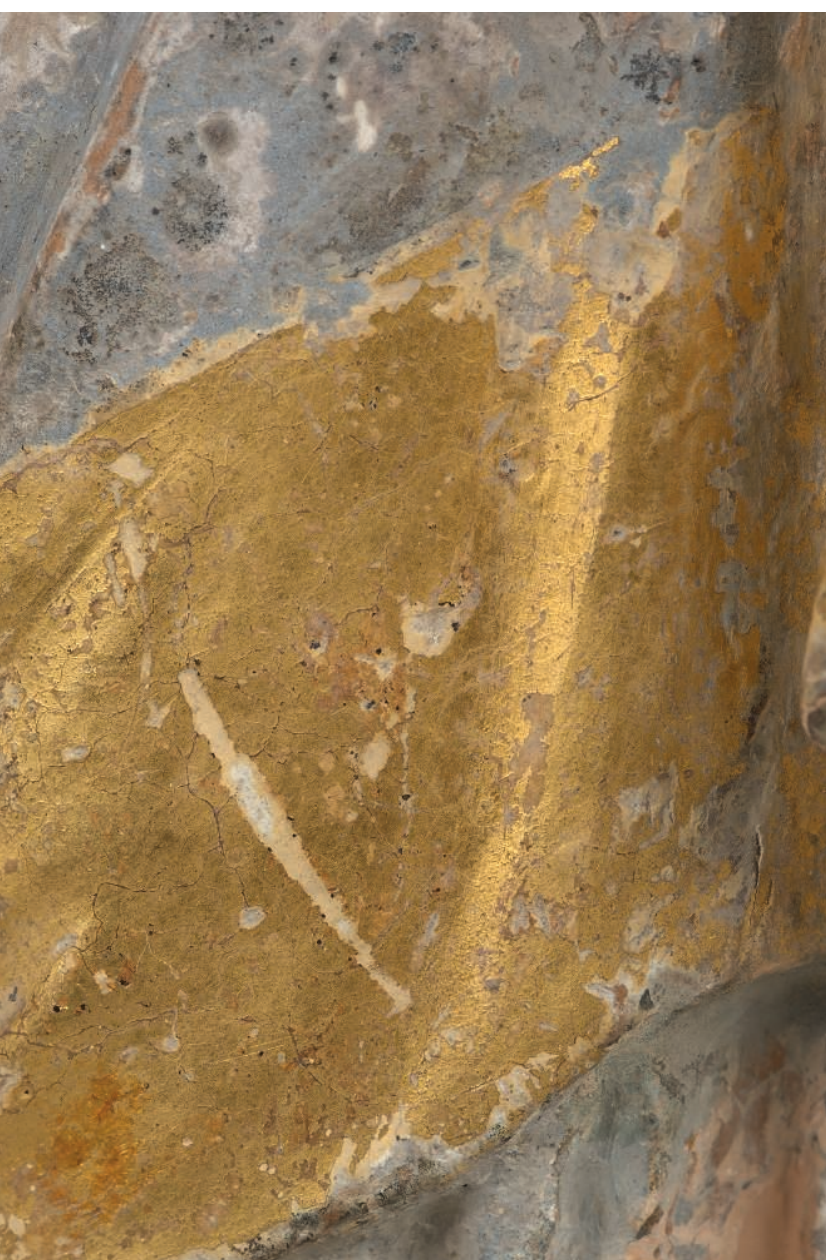

Fig. 1 a. Dame en bleu, Tanagra, vers 330-300, terre cuite peinte et dorée, Louvre inv. MNB 907. Détail : la bordure dorée du bas du manteau. (c) C2RMF/Anne Chauvet.

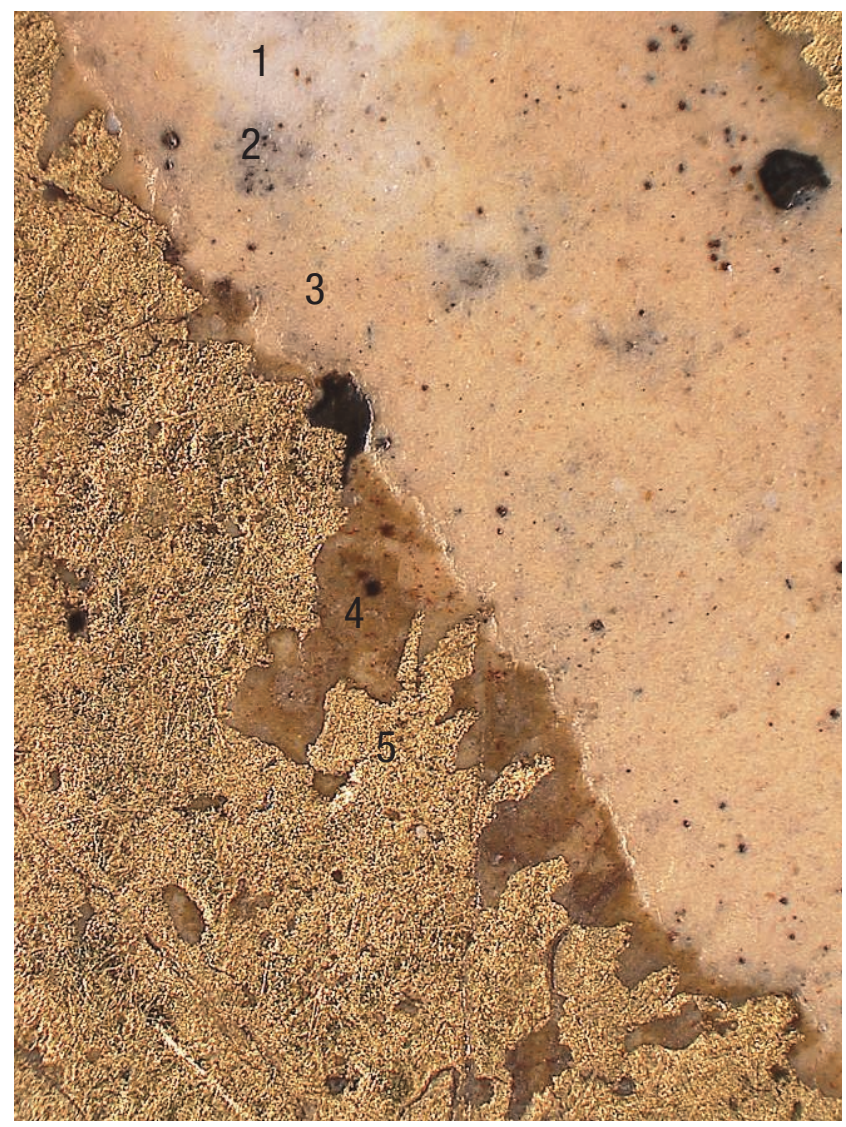

Fig. 1 b. Idem, vue en microscopie numérique (x 140) de la dorure sur le bord de l'entaille moderne. Couche 1 : préparation blanche, couche 2: sous-couche grise, couche 3 : assiette jaune, couche 4 : adhésif, couche 5 : feuille d'or. (c) C2RMF/Brigitte Bourgeois.

\section{Une archéologie historique de la surface}

la face sombre, cachée de l'art, celle du délabrement de la matière, triviale, corruptible, et sur la lutte contre le vieillissement inexorable. Ce n'est plus tant l'original qui nous intéresse, que nous croyons «tenir» au sortir de terre, que son devenir, non plus l'unicité de la création mais la multiplicité des mains, le plus souvent anonymes, qui ont inlassablement lavé, astiqué, recollé et repeint pendant des siècles. S'il est bien question de polychromie originale dans plusieurs articles qui soulignent l'influence, en tous domaines, de la «révolution picturale» du IV ${ }^{\mathrm{e}}$ siècle av. J.-C., il est surtout question de l'attention portée à faire durer l'éclat de la couleur par toute une série de mesures, régulières ou non, mettant en ouvre des savoir-faire précis. Ce qui compte ici n'est donc pas tant l'Urtext que la dynamique de transmission de celui-ci, l'accumulation de gestes déployés autour de l'objet qui peu à peu l'ont sédimenté au cours du temps.
Dans une telle démarche, où l'étude et la conservation actuelle des œuvres du passé sont liées, l'acquisition de données précises, documentées de manière scientifique, passe à l'évidence par l'autopsie de l'objet et par l'auscultation de son épiderme.

Loin d'être une entité de lecture immédiate, donnée au premier regard, la surface, ce «moi-peau» de la sculpture dirait-on en reprenant l'expression forgée par Didier Anzieu ${ }^{4}$, lieu complexe d'échanges entre l'œuvre et son milieu, possède une profondeur physique et historique qu'il convient de sonder. L'idée n'est pas nouvelle. C'était bien dans ce sens qu'en 1989 déjà, Michel Pastoureau recommandait de soumettre l'architecture et la sculpture du Moyen Âge à une véritable «archéologie de la couleur ${ }^{5}$, afin d'établir la chronologie des états et d'étudier le sens d'une évolution sur la 
longue durée. Si la démarche s'est depuis lors considérablement développée pour la période médiévale, comme l'atteste le numéro précédent de Technè ${ }^{6}$, la question est restée encore marginale dans le domaine de l'Antiquité. Pour une raison bien simple: l'étendue de la ruine y est souvent telle que les anciens traitements de surface ont plus ou moins disparu, ne laissant subsister que la forme de marbre blanc mis à nu tel un écorché - ou un inachevé. L'espoir de découvrir des repeints antiques peut donc paraître chimérique ${ }^{7}$. À supposer d'ailleurs que des traces subsistent, ne risquent-elles pas d'échapper à l'attention en raison de leur taille infime? Pour bien faire, l'archéologue de la couleur se doit d'acquérir en quelque sorte ces «yeux de microscope qui percent l'écorce des objets» qu'évoquait un auteur du XVIII ${ }^{\mathrm{e}}$ siècle ${ }^{8}$.

\section{L’apport de la vidéo-microscopie : retour sur un parcours de vingt ans}

Parmi les techniques d'imagerie, la vidéo-microscopie ${ }^{9}$ représente un système d'inspection adapté au travail de terrain, avec ses objectifs variés (zoom ou lentilles fixes) équipant une caméra -autrefois vidéo, maintenant numérique - reliée par un câble souple à une unité centrale permettant de visualiser l'image sur écran (fig. 2-3). L'exploration dynamique de cavités est également rendue possible par l'adaptation d'endoscopes sur le système. Avec le développement du numérique, les appareils récents, dotés de logiciels de micro-mesure, permettent d'accéder au champ immense de l'imagerie 3D de surface et de combiner images et mesures physiques à l'échelle micrométrique.

La publication de différentes études a déjà fait connaître la technique et son apport; mais ce qui manque peut-être est de replacer cette méthode dans l'histoire du développement des sciences du patrimoine au cours des vingt dernières années. Développée initialement pour des applications industrielles et biomédicales, la vidéo-microscopie a commencé à percer dans le domaine de la conservation du patrimoine durant les années 1990. Elle a d'abord servi à affiner le diagnostic des altérations et à contrôler les effets d'interventions de nettoyage. En 1994, Adrian Heritage menait ainsi au Courtauld Institute une étude appliquée au contrôle in situ de peintures murales dans des abbayes anglaises ${ }^{10}$; et, en 1995 , une publication relatait le contrôle du nettoyage d'une sculpture en pierre polychromée ${ }^{11}$. Par la suite, et surtout à partir des années 2000, de nombreux articles ont témoigné d'applications variées, tandis que des synthèses méthodologiques ont également vu le jour ${ }^{12}$.

Au C2RMF, l'adoption de la méthode est également le fait de la restauration et se situe dans ces mêmes années 1994-1995. La filière Archéologie, dont j'étais alors chargée au sein du Service de Restauration des musées de France, était impliquée dans de nombreuses restaurations de marbres antiques, au Louvre et dans d'autres musées. La problématique du nettoyage d'œuvres souvent marquées par un long passé de collectionnisme et de restauration était complexe; leur traitement, sous l'œil attentif de commissions de restauration, suscitait bien des débats. Afin d'éclairer ceux-ci, je veillai à développer, en amont des interventions, le recours à l'imagerie et au contrôle non destructif comme la radiographie de rayons gamma et l'étude microscopique de la surface. Après avoir testé divers appareils, je fis acquérir le premier vidéo-microscope du Service en 1995/6, pour accompagner la campagne de restauration du Gladiateur Borghèse, l'un des grands originaux grecs de l'époque hellénistique conservés au musée du Louvre $^{13}$. D'autres travaux suivirent ${ }^{14}$.

En matière de polychromie antique, domaine encore peu exploré, il était clair que seule la recherche, croisant données historiques et enquête scientifique de terrain, permettrait de progresser dans notre compréhension des états de surface. À la suite d'un premier programme exploratoire que j'avais lancé $^{15}$, la nécessité d'étudier un matériel statuaire demeuré

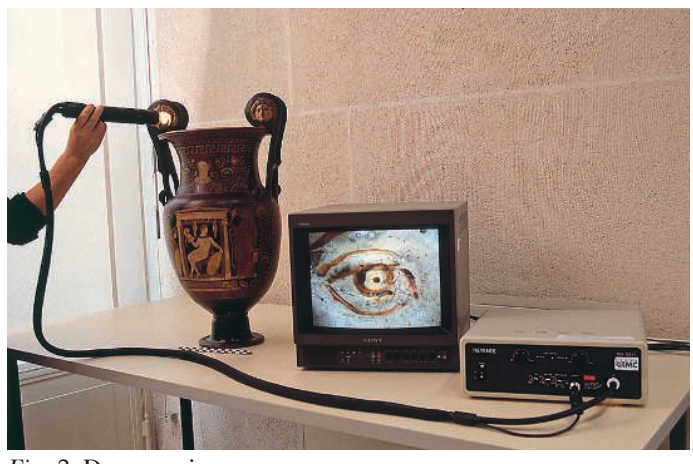

Fig. 2. Du premier appareillage de vidéomicroscopie (Keyence VH-5911) en action dans les ateliers du C2RMF... (C) C2RMF/ Anne Chauvet.

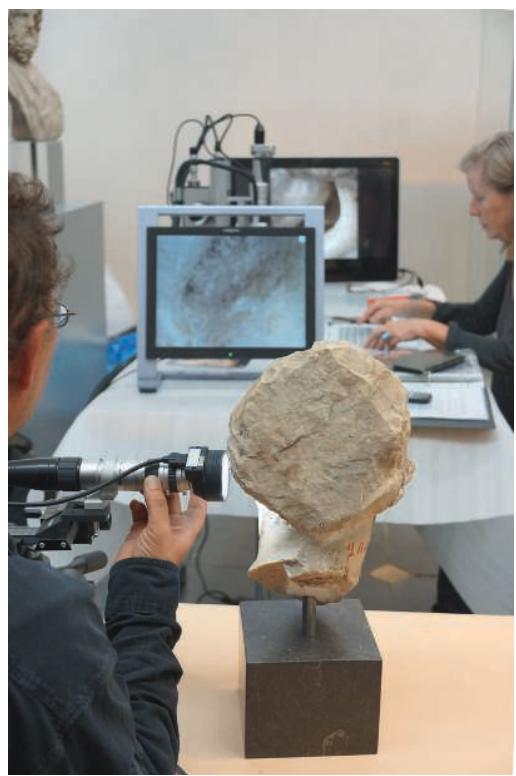

Fig. 3. ....aux derniers travaux sur le terrain: étude du vernis antique en cire d'abeille sur la tête de Bérénice II au Musée royal de Mariemont, avec les appareils Hirox VCR-800 et KH-8700 (microscope 3D). (C) Musée royal de Mariemont/Michel Lechien. 


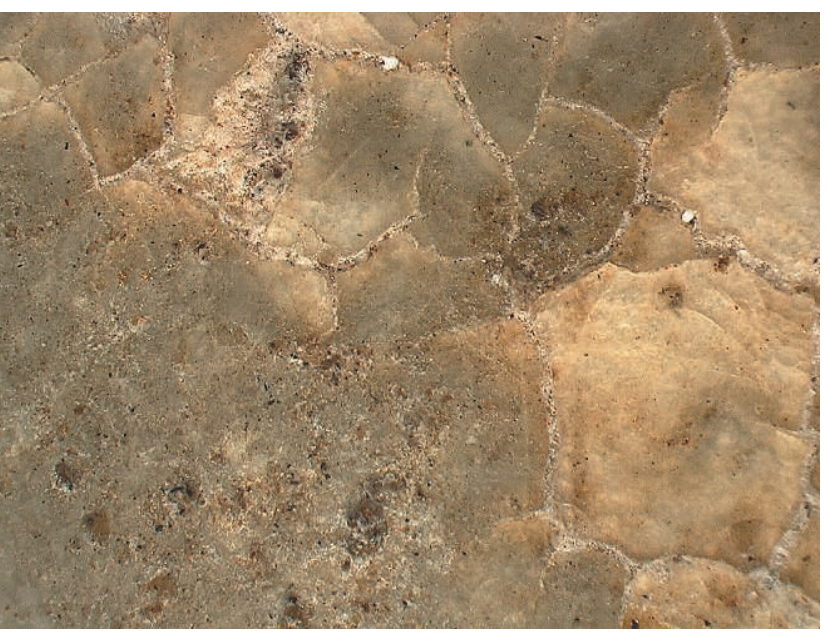

Fig. 4. Tête de Bérénice II (Mariemont, inv. B 264), sur l'oreille droite, vue en microscopie 3D (x 140) d'écailles de cire sous les dépôts d'enfouissement. (C) C2RMF/Brigitte Bourgeois.

plus proche de son état d'exhumation paraissait une évidence. Le professeur Jean Marcadé, membre des commissions de restauration des marbres du Louvre à qui je m'ouvris du projet, m’orienta de suite vers le site de Délos, l'île sainte consacrée à Apollon au cœur des Cyclades, carrefour cosmopolite et prospère entre la koinè hellénistique et la pénétration romaine vers la fin du II $^{\mathrm{e}}$ et le début du I ${ }^{\mathrm{er}}$ siècle av. J.-C. Il avait publié le riche corpus de la sculpture hellénistique de Délos livré par les fouilles de l'École française d'Athènes, et l'un de ses élèves, Philippe Jockey, venait d'en approfondir l'étude technique ${ }^{16}$. Le programme de recherche sur la polychromie des marbres de Délos vit ainsi le jour ${ }^{17}$. Au fil des campagnes sur le terrain (1999-2007), la collaboration, fructueuse, a nourri la publication de plusieurs articles où $\mathrm{Ph}$. Jockey et moi-même avons exposé les résultats produits par la synergie entre l'examen en vidéo-microscopie et l'analyse non invasive en fluorescence X portable ${ }^{18}$.

D’autres découvertes ont été opérées depuis lors dans la mise en évidence, encore plus délicate, des matériaux organiques antiques, qu'il s'agisse par exemple de l'adhésif de la feuille d'or sur une exceptionnelle statuette de Tanagra, la «Dame en bleu » (fig. $1 \mathrm{a}-\mathrm{b}$ ), ou bien du vernis à la cire d'abeille sur la tête en marbre de Bérénice II, au musée de Mariemont (fig. 4, et plus loin p. 75-79). Les implications de telles études sur la politique de conservation, et notamment sur l'extrême prudence à observer en matière de nettoyage des surfaces antiques, sont évidemment considérables ${ }^{19}$. Une fois de plus, recherche et restauration ont ici étroitement partie liée.

\section{Le contenu du numéro}

L'essentiel de l'enquête porte sur le monde grec du IV siècle, de l'époque hellénistique et romaine (Grèce, Grande-Grèce, Égypte, Chypre). Cependant, pour inscrire davantage le phénomène de la conservation matérielle de la sculpture dans sa longue durée, deux contributions relatives à l'Égypte pharaonique ont été incluses. La dernière partie du numéro aborde la destinée ultérieure de la sculpture, détruite largement durant l'Antiquité tardive avant de «renaître » à l'époque moderne, que ce soit via l'étude ou via la tentative d'artistes, tel Gérôme, de renouer avec l'art des Anciens. Faute de place, on n'a pu intégrer, parmi les facteurs ayant contribué au développement de la restauration dans l'Antiquité, le rôle du collectionnisme, pourtant si important dans le cadre des monarchies hellénistiques, puis dans le monde romain.

Concernant la méthode, il a semblé important de bâtir une démonstration interdisciplinaire qui s'appuie à la fois sur l'établissement de faits matériels précis (via des études de cas) et sur une remise en contexte historique plus large. Celle-ci permet de prendre un certain recul par rapport à nos grilles de lecture modernes, aisément inadaptées et anachroniques. L'essai de synthèse qu'établit dans les années 1950 un historien de la restauration comme Cagiano de Azevedo critiquait la «désinvolture» de la pratique antique par rapport à l'artiste créateur ${ }^{20}$. Mais l'obsession de l'authorship, propagée en histoire de l'art occidental depuis Vasari, a fait son temps ${ }^{21}$; et les mots «statue», «artiste», «polychromie », au sens où nous les entendons, renvoient à des notions largement construites par l'histoire de l'art et la muséologie modernes, étrangères aux réalités grecques ${ }^{22}$. Il vaut donc mieux procéder autrement et repartir des catégories mentales des Anciens ${ }^{23}$.

Dans un domaine comme celui-ci, la question du vocabulaire est évidemment épineuse, et l'on trouvera selon les auteurs des définitions différentes pour un mot tel que restauration. Seule une connaissance approfondie du contexte (volonté du ou des commanditaires, modalités de l'intervention technique, maintien ou non d'un état vieilli de l'œuvre originelle - ce qui n'est pas exclu dans certains cas) pourrait faire décider s'il faut par exemple traduire épiskévè par conservation, ou réparation ou restauration; or celle-ci nous fait défaut en général. L'impression d'une certaine confusion lexicale pourra donc peut-être surgir de la lecture des articles. Mais si nous comparons l'état de la question avec les réalités contemporaines, n'est-ce pas aussi bien souvent le cas? Et ne connaissons-nous pas l'écart qui peut exister entre le discours qui habille la pratique et celle-ci?

Je conclus en remerciant chaleureusement tous les auteurs qui, en dépit d'un calendrier chargé, ont accepté de participer à cette aventure éditoriale, notamment mes collègues grecques, Hariclia Brecoulaki et Evridiki Leka; sans elles, ce retour aux sources n'aurait pu prendre tout son sens. D'autres m'ont également aidée qui ne figurent pas au sommaire. À Jackie Pigeaud, source d'inspiration dans ses écrits et dans l'entretien que nous avons eu, à Béatrice Chemama, Dominique Robcis, aux relecteurs du comité de rédaction, en particulier Violaine Jeammet et Stéphanie Deschamps-Tan, à Cécile Binet, MarieLiesse Boquien, Joëlle Crétin, Gabrielle Vitali et Anne Terral, assistante infatigable, j'adresse également mes vifs remerciements. Je dédie enfin ce volume à la mémoire de Jean Marcadé. 
Notes

1. Berducou, 2010.

2. Voir notamment le séminaire de M. Carastro (EHESS) et, en 2012, le colloque «Agalma ou les figurations de l'invisible» (Anhima -UMR 8210). Également Figura XIV. Construire le divin en images, Presses Universitaires de Rennes, à paraître.

3. Voir en dernier lieu l'exposition de la Ny Carlsberg Glyptotek à Copenhague.

4. Anzieu, 1985.

5. Pastoureau, 1989 , p. 210

6. $\mathrm{N}^{\circ} 39,2014-1$, La polychromie des sculptures françaises du Moyen Âge, sous la dir. de S. Deschamps-Tan, P.-Y. Le Pogam, C. Raynaud.

7. Comme le dit par exemple Marina Prusac, From Face to Face. Recarving of Roman Portraits and the Late-Antique Portrait Arts, Brill, Leiden, London, 2011, p. 4. Pour des repeints et redorure en sculpture romaine, voir M. Abbe dans Circumlitio, 2010, et P. Liverani dans le volume qu'il a dirigé avec U. Santamaria, Diversamente bianco. La policromia della scultura romana, 2014.

8. G. Gauchat, Lettres critiques ou analyses et réfutations de divers écrits modernes contre la religion, Genève, 1755-1759, cité par Abramovici, 1998, p. 388

9. Orthographiée en français et en anglais avec ou sans trait d'union.

10. Voir de cet auteur An evaluation of the potential of video microscopy in the examination, recording and monitoring of wall paintings in situ, dissertation, Courtauld Institute, 1994, puis Imaging dynamic processes in conservation: timelapse video microscopy with on-line data acquisition and direct image annotation, 1995. Getty Conservation Institute.

11. Couronnement de la Vierge de Terlano, à Padoue, voir l'article de Stampfer, Dapunt et Driussi dans Biscontin G., Driussi G., La pulitura delle superfici dell'architettura: atti del convegno di studi, Bressanone, 3-6 luglio 1995, Padova, p. 507-520.

12. Voir par exemple le nettoyage laser de la Porte du Paradis du Baptistère de Florence (Siano, Salimbeni, 2001) et l'article méthodologique de Stanley, 2000. Hors secteur patrimonial, manuels tels que Digital microscopy: a second edition of video microscopy, Elsevier Academic Press, 2003.

13. Voir Bourgeois, Pasquier, 1997, p. 20, contrôle de la surface du marbre avant et après nettoyage.

14. Par exemple, Blanchegorge, Bourgeois, de Drée, Drouet, «Dévoiler l'antique", Technèn ${ }^{\circ}$ 13-14, 2001 (détection de couleur antique ayant guidé des choix de dérestauration sur une coupe attique); Bourgeois, 2007 (polychromie des figurines en terre cuite, dans le cadre de l'exposition Tanagra, Louvre, 2003).
15. Recherches sur les traitements de surface des marbres antiques, Service de Restauration des Musées de France, 19961997, financé par la Mission de la Recherche et de la Technologie, participants: B. Bourgeois (pilote), J. Délivré, Néguine Mathieux, et laboratoires de l'Université de Reims (V. Barbin), CNEP (D. Fromageot) et LETIAM (J. Bléton, A. Tchapla).

16. Marcadé 1969, et thèse de doctorat de Ph. Jockey, ancien membre de l'École française d'Athènes, Techniques et ateliers de sculpture à Délos à l'époque hellénistique, Université Paris X - Nanterre, 1993.

17. Avec le soutien de J.-P. Mohen, F. Dijoud (direction du C2RMF), et de R. Etienne (directeur de l'EFA) que j'ai plaisir à remercier ici.

18. Collaboration d'A. Karydas et $\mathrm{H}$. Brecoulaki. Voir Bourgeois, Jockey, 2007 (avec bibliographie antérieure) et 2010 notamment

19. Bourgeois, 2012.

20. Cagiano, 1952 , p. 58 . Il y aurait beaucoup à dire aussi sur l'opinion selon laquelle l'Antiquité n'aurait connu que la réparation (primat de la fonction de l'objet) ou la réfection/remise au goût du jour (primat de la volonté d'embellir), et non pas la restauration.

21. Voir notamment ce qu'en dit Spivey, 1996 , p. $14-15$.

22. Rolley, 1994, chap. 1 «Qu'est-ce qu'une statue?» et chap. 2 «Le sculpteur et son public».

23. Comme l'ont fait notamment Carastro, 2008, et Grand-Clément, 2013, pour la couleur.

\section{Bibliographie sélective}

Abramovici J.-Ch., 1998, «Entre vision et fantasme: la réception en France des «curieux microscopes» (1660-1800)» dans Nicole Jacques-Chaquin, Sophie Houdard (ed.), Curiosité et Libido sciendi de la Renaissance aux Lumières, Fontenay-aux-Roses, ENS Éditions, tome 2, p. 371-392.

Anzieu D., 1985, Le moi-peau, Paris, Dunod.

Berducou M., 2010, «Bref voyage dans le passé du passé. Continuité et ruptures dans l'histoire de la conservation du patrimoine", Technè 32, Une perfection dangereuse. La restauration des vases grecs, de Naples à Paris, XVIII $-\mathrm{XIX}^{e}$ siècles, p. 11-17.

Bourgeois B., 2012, «Marbre blanc, taches de couleur. Polychromie et restauration de la sculpture grecque » dans Kairis P.-Y., Sarrazin B., Trémolières F.,
La restauration des peintures et des sculptures. Connaissance et reconnaissance de l'œuvre, Paris, Armand Colin, p. 25-42.

Bourgeois B., 2007, «Pratiques artisanales de la couleur. Enquête sur la polychromie des Tanagréennes » dans Jeammet V. (dir.), Tanagras. De l'objet de collection à l'objet archéologique, Louvre/ Picard, Paris, 2007, p. 81-89.

Bourgeois B., Jockey Ph., 2010, "The Polychromy of Hellenistic Marble Sculpture in Delos" dans Brinkmann V., Primavesi O., Hollein M. (éd.), CIRCUMLITIO. The Polychromy of Antique and Mediaeval Sculpture, Liebieghaus, Frankfurt am Main, Hirmer Verlag, Munich, p. 224-239.

Bourgeois B., Jockey Ph., 2007, «Le marbre, l'or et la couleur » dans DescampsLequime S., (éd), Peinture et couleur dans le monde grec antique, Louvre, Paris, 5 Continents, Milan, p. 162-191.

Bourgeois B., Pasquier A., 1997, Le Gladiateur Borghèse et sa restauration, Louvre, Paris.

Cagiano de Azevedo M., 1952,

«Conservazione e restauro presso i Greci ed i Romani », Boll. dell' Istituto Centrale per il Restauro, 9-10,1952, p. 53-60.

Carastro M. (dir.), 2008, L’Antiquité en couleurs: catégories, pratiques, représentations, Grenoble.

Ebbinghaus S. (guest editor), 2011, SOURCE: Notes in the History of Art, vol. XXX $\mathrm{n}^{\circ} 3$, spring 2011, Special issue: Superficial? approaches to painted sculpture.

Grand-Clément A., 2011, La fabrique des couleurs. Histoire du paysage sensible des Grecs anciens, VIII ${ }^{e}$-début du $V^{e}$ s. av. n. è., Paris, éditions de Boccard.

Marcadé J., 1969, Au musée de Délos. Étude sur la sculpture hellénistique en ronde-bosse découverte dans l'île, BEFAR fasc. 215 EFA, Paris, éditions de Boccard.

Østergaard J. S., Nielsen A.M. (eds.), 2014, Transformations: Classical Sculpture in Colour, Ny Carlsberg Glyptotek, Copenhagen.

Pastoureau M., 1989, «L'Église et la couleur, des origines à la Réforme », Bibliothèque de l'école des Chartes, tome 147, p. 203-230.

Rolley C., 1994, La sculpture grecque, 1, Des origines au milieu du $V^{e}$ siècle, Paris, Picard éditeur.

Spivey N., 1996, Understanding Greek Sculpture: ancient meanings, modern readings, London, Thames and Hudson.

Stanley T., 2000, "Digital video microscopy: a practical visual analysis technique for the conservator", Journal of the American Institute for Conservation, 39, 2, p. 205214. 